\title{
B2 - Estudo clínico retrospectivo da alfataliglicerase no Hemorio
}

Vivian Rotman ${ }^{1}$; Patrícia M. N. de Oliveira ${ }^{1}$; Hugo Defendi ${ }^{1}$; Tatiana Guimarães de Noronha ${ }^{1}$; Deborah Araújo da Conceição ${ }^{1}$; Janaína Reis Xavier ${ }^{1}$; Maria de Lourdes de Sousa Maia'; Renata de Souza Cravo²

1 - Bio-Manguinhos/FIOCRUZ;

2 - Instituto Estadual de Hematologia Arthur Siqueira Cavalcanti /Hemorio.

\section{Introdução:}

O Hemorio é a unidade de referência no tratamento de doença de Gaucher (DG) no Rio de Janeiro/Brasil. Bio-Manguinhos vem liderando processos de desenvolvimento conjunto de medicamentos biológicos, incluindo a transferência de tecnologia do biofármaco alfataliglicerase. A alfataliglicerase é uma terapia de reposição enzimática (TRE), produzida por um método novo, baseado na cultura de células vegetais (cenoura) e tem sido utilizado no Brasil desde outubro de 2010, no tratamento da DG.

\section{Objetivo:}

Avaliação dos parâmetros clínicos e laboratoriais de eficácia e de segurança da alfataliglicerase.

\section{Metodologia:}

Estudo retrospectivo, com base na avaliação de prontuários de pacientes com DG tipo 1, tratados com alfataliglicerase no Hemorio. Os parâmetros foram avaliados antes e durante a utilização de alfataliglicerase. Para análise de eficácia, os pacientes foram divididos em 2 grupos: grupo A $(n=13)$, dos que iniciaram alfataliglicerase em 2010 e utilizam até hoje e grupo $B(n=18)$, de pacientes que usaram a alfataliglicerase mas não utilizam atualmente. Para o grupo $\mathrm{B}$ a eficácia foi avaliada apenas para aqueles que utilizaram o medicamento por um período mínimo de 12 meses ( $\mathrm{n}=9$ ). Avaliação de segurança foi feita em conjunto (grupos $\mathrm{A}+\mathrm{B})$.

\section{Resultados:}

No grupo A, o tempo médio de uso da alfataliglicerase por participante foi de $3,6( \pm 0,05)$ anos. O genótipo mais comum foi o L444P/N370S, presente em $46 \%$ dos pacientes. Ao comparar a evolução pareada do fígado e baço, observou-se que $46 \%$ dos participantes mantiveram os dois parâmetros estáveis. A 
média da hemoglobina na avaliação de 1 ano antes do início da alfataliglicerase foi de $14 \mathrm{~g} / \mathrm{dl}(+/-1,5)$ e a média da medida mais recente (2013-2014) durante o uso da alfataliglicerase foi de $13,4 \mathrm{~g} / \mathrm{dl}(+/-2,2)$. Não houve variação significativa entre os valores médios de plaquetas, antes e durante o uso de alfataliglicerase. No grupo B, o tempo médio de uso da alfataliglicerase por participante foi de 3,6 $( \pm 0,05)$ anos. O genótipo mais comum foi o N370S presente em $39 \%$ dos pacientes. Com relação ao fígado, nos 9 pacientes com esta avaliação, 6 (67\%) mantiveram estável o volume hepático à palpação, 2 (22\%) apresentaram aumento e em 1 (11\%) houve diminuição. Em relação ao baço, 4 pacientes (44\%) eram esplenectomizados. Entre os $5 \mathrm{em}$ que foi analisado o volume esplênico, 2 (40\%) mantiveram este parâmetro estável, em 1 (20\%) houve aumento e em 2 (40\%) diminuição. No que concerne medidas de $\mathrm{Hb}$ e plaquetas, não foram observadas alterações significativas nos valores antes e durante o uso de alfataliglucerase. Entre os eventos adversos esperados, os mais frequentes foram os musculoesqueléticos e de tecido conjuntivo. Em 3 pacientes $(9,7 \%)$ houve necessidade de interrupção do tratamento por reação infusional.

\section{Conclusão:}

A alfataliglicerase apresentou perfis de eficácia e segurança favoráveis.

Palavras-Chave: Alfataliglicerase; Doença de Gaucher; Estudo clínico retros-
pectivo 